\title{
THE SPATIAL AND TEMPORAL BEHAVIOUR OF THE LOWER STRATOSPHERIC TEMPERATURE OVER THE SOUTHERN HEMISPHERE: THE MSU VIEW. PART II: SPATIAL BEHAVIOUR
}

\author{
M. ALEJANDRA SALLES ${ }^{1}$, PABLO O. CANZIANI*,2 and ROSA H. COMPAGNUCCI ${ }^{3}$ \\ Departamento de Ciencias de la Atmósfera, Facultad de Ciencias Exactas y Naturales, Universidad de Buenos Aires/CONICET, \\ Buenos Aires, Argentina \\ Received 24 December 1999 \\ Revised 30 August 2000 \\ Accepted 31 August 2000
}

\begin{abstract}
Monthly lower stratosphere temperature anomalies in a layer centred about $70 \mathrm{hPa}$, from the MSU data set are analysed for the period 1979-1997. T-mode approach principal component analysis (PCA) is used in order to obtain the leading spatial anomaly patterns and their sequences of occurrence throughout the period under study. Five principal components (PCs) are significantly different from the spatial distribution of noisy data. The patterns given by the PC scores represent ten typical spatial anomaly patterns: five correspond to the direct mode, that is to say anomaly fields with the same sign as the PC score patterns, and five have the opposite sign. The first three PCs represent simple spatial temperature anomaly distributions, with zonal wave 0 to wave 2 wave structures. The following significant PCs, orders four and five, display a more complex spatial behaviour, with wave 3 wave structures. The first two PC's frequency distribution in time, given by the PC loadings time series, do not show noticeable changes throughout the period analysed. The remaining three PCs show changes in their frequency of occurrence that might be associated with the negative trends in the lower stratosphere temperature, as well as to the other different features observed in the real temperature anomaly time series for the grid points in the Southern Hemisphere. The latter are studied with the PCA in the S-mode approach in the companion paper (Compagnucci $e t$ al., 2001. International Journal of Climatology 21: 419-437). Copyright (C) 2001 Royal Meteorological Society.
\end{abstract}

KEY WORDS: stratosphere; temperature; variability; MSU

\section{INTRODUCTION}

Compagnucci et al. (2001) (the companion paper to this, from now on Part I) applied multivariate analysis tools to the study of the temporal behaviour of the monthly mean lower stratospheric temperature anomalies over the Southern Hemisphere, as measured by the Microwave Sounding Unit (MSU). This family of satellite-borne sensors provides a continuous series of temperature measurements spanning 19 years, at various heights in the troposphere and the lower stratosphere, with high spatial resolution and global coverage. In particular channel 4 retrievals (from now on referred to as T4) correspond to a deep layer between 150 and $50 \mathrm{hPa}$, with a maximum contribution from the field in the vicinity of $70 \mathrm{hPa}$ being used. This corresponds to a lower stratospheric layer, except near the Equator, where contributions from the upper troposphere are included in the vertical integration of T4 products. For more details about the MSU the reader is encouraged to refer to the second section of Part I and references therein.

\footnotetext{
* Correspondence to: Departamento de Ciencias de la Atmósfera y los Océanos, FCEN-UBA, Pabellón II-2do Piso, Ciudad Universitaria, 1428 Capital Federal, Argentina; e-mail: canziani@rosario.at.fcen.uba.ar

${ }^{1}$ E-mail: salles@at.fcen.uba.ar

${ }^{2}$ Also at: Laboratorio de Investigaciones de Sistemas Ecológicos y Ambientales, Facultad de Ciencias Naturales y Museo, Universidad Nacional de La Plata, Argentina.

${ }^{3}$ E-mail: rhc@at.fcen.uba.ar
} 
A principal component analysis (PCA) method in the S-mode approach was applied in Part I to determine the main characteristics of the temporal variability and areas of influence of these modes. In that study the temporal variability was studied for time series at each point on a $2.5^{\circ} \times 2.5^{\circ}$ grid in the region of interest, i.e. the Southern Hemisphere. Thus, both the characteristic time series for clusters of points and the areas where these time series are representative of the time behaviour were determined. However, this analysis did not allow the determination of the main spatial characteristics of the field under study, nor the recurrence of such spatial patterns. Indeed, in the S-mode analysis, for each of the PCs, the significant correlation clusters of the PC loadings maps represent regions where a given time series is significant, but there is no information about what happens in the rest of the area under study. At a given time, the actual field will result from the combination of the particular states of the different dominant temporal modes in each region or cluster within the area of study, at that particular time. Thus, the determination of the main spatial patterns in the MSU T4 data set is necessary to understand the behaviour of the temperature anomalies in their spatial variability. Indeed a complete image of the spatial and temporal variability of the lower stratospheric temperature cannot be obtained without a study of its spatial distribution.

In order to obtain such information, another approach to the PCA must be applied, known as the T-mode approach (Cattell, 1952). In consequence, the PC T-mode methodology was applied to the same data set used in Part I, so as to identify the main spatial characteristics of the temperature anomaly fields. The second section presents a brief overview of the results obtained in Part I. Since the data have already been described in Part I, and the main aspects of PCA discussed there too, only the specific details pertaining to the T-mode approach will now be presented in the third section. It must be noted that the T-mode methodology has not enjoyed such a widespread use as the S-mode, despite the benefits that could be obtained by the joint use of both approaches, as shown in this work. The fourth section presents the results of the analysis and the fifth section will discuss these results together with those obtained using the S-mode approach. The fifth section presents the main conclusions reached in this and the companion paper.

\section{A BRIEF OVERVIEW OF THE S-MODE ANALYSIS RESULTS}

The results of Part I have been useful in determining the temporal behaviour of the lower stratosphere for the last 19 years, and how different temporal behaviours are affecting the variability at different regions of the hemisphere. With those results it was even possible to infer what processes could be affecting the temporal behaviour.

Basically five principal components (PCs) were necessary to describe the main temporal patterns (PC scores), with variability at different time scales, of the lower stratosphere over the Southern Hemisphere. A sixth PC describes a more complex behaviour. The main Southern Hemisphere clusters of grid points with similar time series patterns, yielded by the PC loadings, i.e. correlation maps are, by order of described variance:

(i) a tropical/subtropical quasi-zonal ring;

(ii) a circular region above Antarctica;

(iii) a dipole at mid to high latitudes to the south of Africa and to the west of the Bellingshausen Sea in the Pacific Ocean;

(iv) a dipole perpendicular to the previous one, over the Weddell Sea and Patagonia, on the Atlantic Ocean side, and from New Zealand southward on the Pacific Ocean sector;

(v) a ring over the Equator and a subtropical to temperate latitudes region, particularly over the Pacific.

Only the first PC showed distinctly the signature of the impact of volcanic eruptions upon the stratosphere, while the second described the influence of the cooling over Antarctica, most probably due to ozone depletion. The dipoles describing the third and fourth PCs showed an anti-phase behaviour 
between the Atlantic/Indian Ocean south of Africa and Pacific Ocean sectors and, less clearly, between the Atlantic and South Pacific sectors, respectively. Finally, the fifth PC described the behaviour of the Quasi-Biennial Oscillation (QBO) over the Equator. A cooling trend of varying magnitude was observed in all cases, except over New Zealand and the adjacent area of influence of the fourth PC score time series. All these trends, which as a result of the S-mode analysis are obtained in a longitudinal distribution, are in good agreement with published zonal results (Angell, 1988; Ramaswamy et al., 1996; Graf et al., 1998).

\section{A BRIEF INTRODUCTION TO THE PCA T-MODE METHOD}

The definitions introduced in Part I will now be considered in terms of the T-mode approach (Cattell, 1952). When this approach is applied, the spatial fields of the variable under study, at a given time, are defined as the statistical variables. Preisendorfer (1988) introduced the term 'snapshot' to describe the instantaneous or time-mean field (e.g. the field data at a given time or day or the mean field over a given time period) over the complete area under study, at a given time. The domain is now the time and the statistical observations are the different points in space included in the 'snapshot', or instantaneous field. In other words, the main spatial patterns of the temperature anomaly field are now obtained. The associated time series shows when the particular pattern is present and how significant its contribution is to the real field at a particular time. Hence, the T-mode is not comparable to the S-mode but complementary. It must be stressed that the results from one cannot, in general, be reproduced through the mathematical manipulation of the other. Furthermore, if the S- and T-modes were to yield apparently similar results this would imply a simple linear relationship between space and time, precluding any other effects, such as dispersion, which takes place over time due to the non-linearity of real systems.

The use of this method is far less common in literature than the S-mode approach. Richman (1983), Drowsdowsky (1993), Huth (1993), Compagnucci and Salles (1997), Compagnucci and Vargas (1998) and Compagnucci et al. (1999) are among the few who have used the T-mode analysis.

In this study, the variables are the spatial field or 'snapshot' of the mean monthly temperature anomaly $(n=228)$. The statistical observations are now grid points $(m=5148)$. The patterns yielded by the PC scores are the spatial fields showing the principal or leading types of anomalies. These describe the observed spatial variability. The PC loading are the time series, which represent the correlation between the PC spatial patterns and each of the monthly mean real anomaly fields. In this case, the variance explained by each component determines the degree of significance of each spatial mode in the temporal domain. As was the case before, when the values of the PC loading are small and/or non-significant, i.e. which belong to the hyperplane, this spatial pattern does not correspond to the structure of the temperature anomaly field at that given time (Richman and Gong, 1999). In this case the absolute values in the time series which are within \pm 0.3 are considered non-significant, i.e. they belong to the hyperplane. This value is within the range proposed by Richman and Gong (1999). This means that for those times the particular derived spatial pattern under consideration is not representative of the structure of the field.

\section{THE SPATIAL BEHAVIOUR OBTAINED USING THE T-MODE APPROACH}

The spatial structure of the temperature anomalies can be described in terms of the first five PC scores or spatial maps. They explain $65.57 \%$ of the total variance of the sample. This can be observed in the Lev diagram (Figure 1) which displays the $\log _{10}$ of the eigenvalues versus the order of the corresponding PCs. The plot shows that the first five components are distinct from the slope of the 'tail', which corresponds to noisy values. The sixth model is already in the slope of the tail, and it can only explain $3.4 \%$ of the variance. Hence, it is not considered here. It is interesting to note that the first five modes in the T-mode approach describe less variance than was the case for the S-mode, the difference being that in this case this value corresponds to the spatial variance which obviously has different characteristics from the temporal one. 


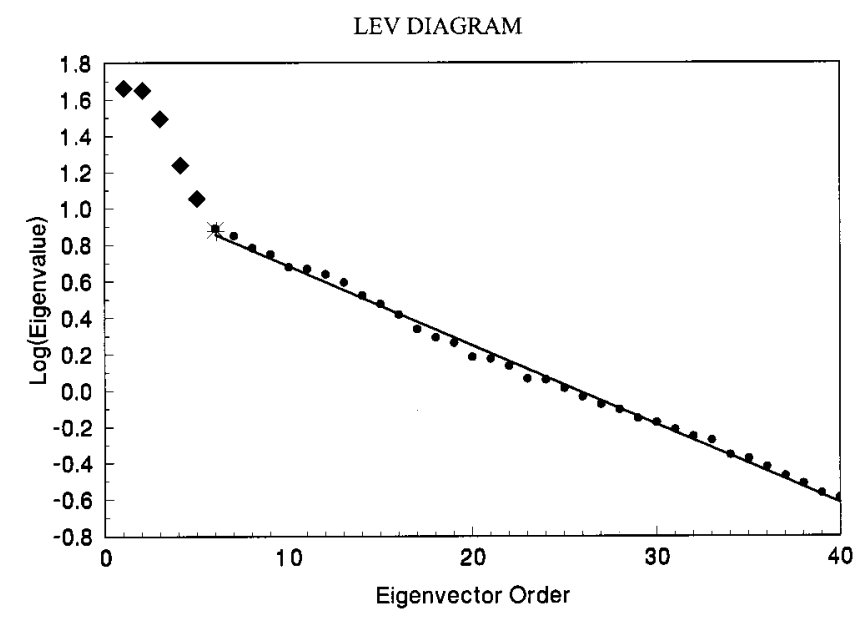

\begin{tabular}{|c|c|c|c|c|}
\hline PC & Eigenvalue & $\begin{array}{c}\text { \% Var. Direct } \\
\text { Mode }\end{array}$ & $\begin{array}{c}\text { \% Var. } \\
\text { Inverse Mode }\end{array}$ & $\begin{array}{c}\text { \% Cumulative } \\
\text { Variance }\end{array}$ \\
\hline $\mathbf{1}$ & 45.60 & 9.88 & 10.12 & 20.00 \\
\hline $\mathbf{2}$ & 44.06 & 8.48 & 10.84 & 39.32 \\
\hline $\mathbf{3}$ & 31.52 & 7.60 & 6.22 & 53.14 \\
\hline $\mathbf{4}$ & 17.14 & 4.50 & 3.02 & 60.65 \\
\hline $\mathbf{5}$ & 11.21 & 3.07 & 1.85 & 65.57 \\
\hline
\end{tabular}

Figure 1. Lev diagram $\left(\log _{10}\right.$ of eigenvalue versus eigenvector order): the first five PC-eigenvalues are highlighted with diamonds, the sixth eigenvalue with a star and the fitted linear trend for the noise tail is shown. The table displays the eigenvalues, the corresponding percentages of the explained variance by the direct mode, by the inverse mode and the cumulative variance for the first six PCs

The results for the T-mode PCs are shown in the subsequent plots. In all cases the PC score spatial map is presented, together with a composite field for all the real fields that fall within the hyperplane, described by that particular PC and the PC loading correlation time series. Separate composite plots are shown for direct and inverse modes. It must be noted that the PC score fields are non-dimensional, while the real fields are in degrees Kelvin. The standardization of the real fields does not result in major modifications to the maps and hence the 'raw' composite maps, obtained without standardizing them by the standard deviation (S.D.) of the sample, were included in the figures. The spectra corresponding to the PC loading time series, calculated as in Part I, are only shown for the first two PCs. This is due to the fact that for subsequent PCs a major part of the correlation values fall outside the hyperplane. Consequently the fewer values remaining outside the hyperplane can be viewed rather as more or less repetitive events and not as continuous occurrences.

\section{1. $P C 1$}

The first PC score (Figure 2), which explains $20 \%$ of the variance, has a dipole anomaly structure with the largest positive and negative values located at mid to high latitudes and very small or null values at subtropical and tropical latitudes. The axis of the dipole is aligned along $60^{\circ} \mathrm{E}-120^{\circ} \mathrm{W}$, and for all practical consequences can be taken to be centred over the South Pole. The most important anomalies are found at approximately $60^{\circ} \mathrm{S}$.

The direct mode shows positive values Southeast of Africa and negative values over the Southern East Pacific. This mode corresponds to $9.8 \%$ of the total observed variance. The corresponding composite map, shown in Figure 2(b), as an excellent agreement between the composite field and the PC score plot. The best correlation with the PC score pattern, as well as with the temperature anomaly field is observed in June 1992 (not shown). Note that the temperature anomaly range of this composite field is of the order of 1.5-3 K. The PC loading time series (Figure 2(d)) shows that a number of months from the sample share this mode, with high positive correlation values. There are at least eight occurrences where the 
a)

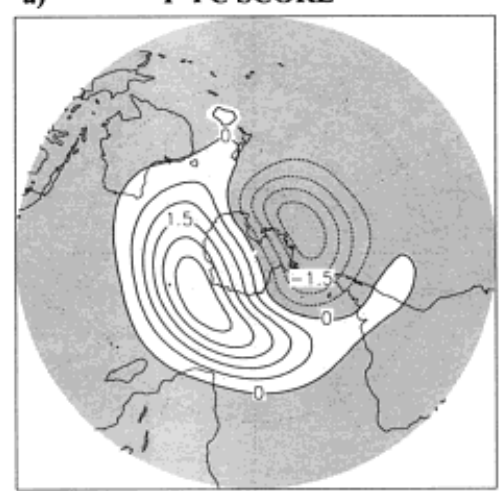

b)

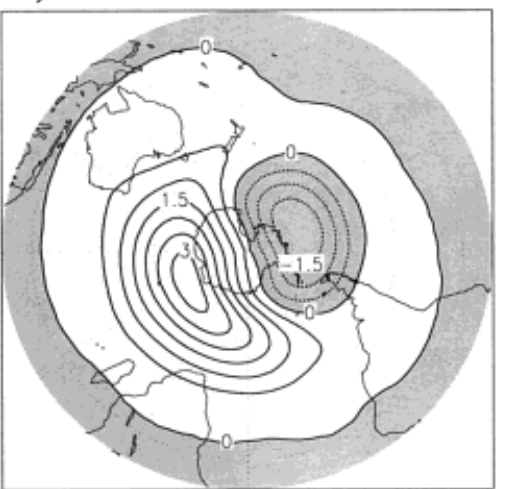

c)

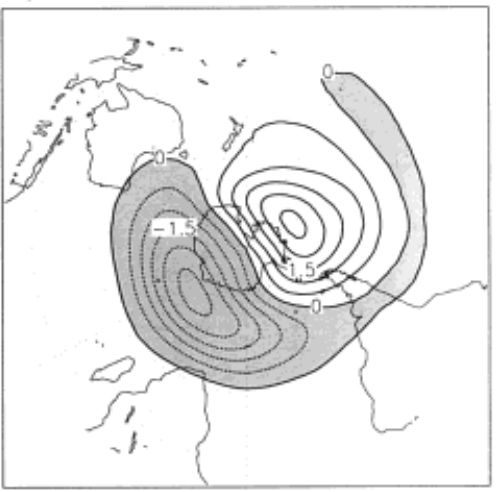

d)

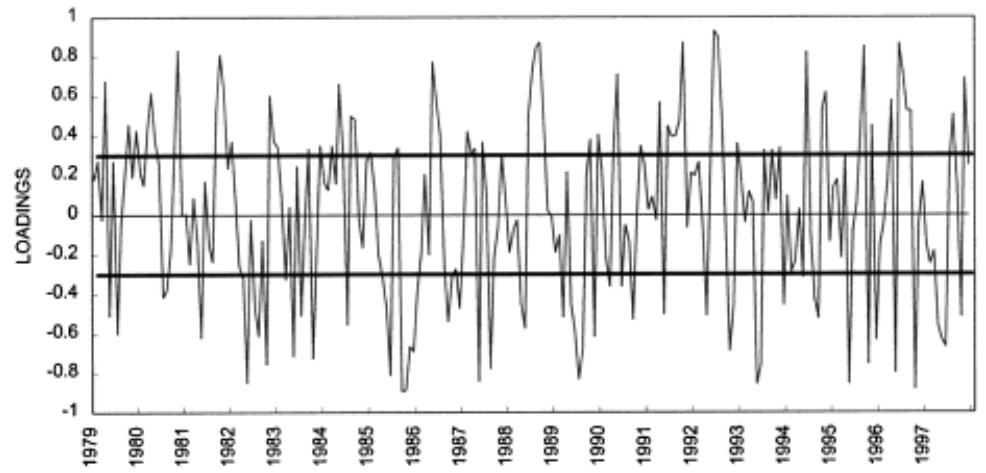

e) PC LOADING SPECTRUM

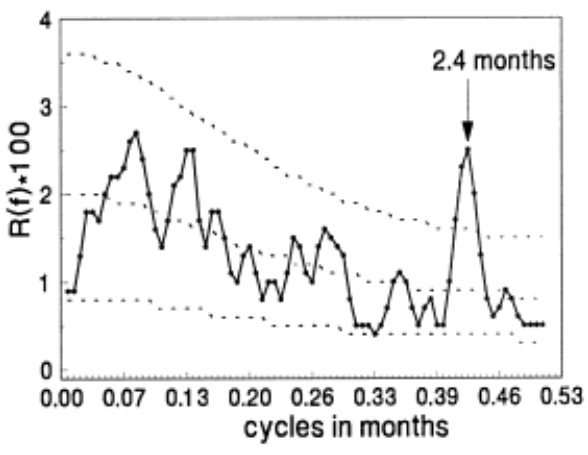

Figure 2. Main aspects of the first PC: (a) PC score spatial pattern, contour values are every 0.5 with shaded areas for negative score values; (b) real field composite for direct mode events, in K; (c) real field composite for inverse mode events, in K; (d) PC loading time series and the hyperplane levels for \pm 0.3 ; (e) normalized spectral analysis according to Tukey with a Parzen window given as $R(f) \times 100$ versus cycles/month (full line) and the $90 \%$ confidence level considering theoretical Markov process. Significant peaks are highlighted

correlation is higher than 0.8 ; that implies that this mode describes more than $64 \%$ of the variance of the corresponding fields. The occurrence of these fields appears to be clustered about June and September, October and November.

The inverse mode has positive anomalies over the Pacific and negative ones on the other side of Antarctica. This inverse mode represents about $10.2 \%$ of the total spatial variance, i.e. a slightly larger occurrence of such fields with respect to the direct mode. There are at least nine times when the observed field correlates at least at the -0.8 correlation value with the first PC score pattern. These high negative correlation events are well-clustered about May and June for the 19-year sample, though there are some occurrences in September and November. Inspection of the inverse composite field (Figure 2(c)) is also in excellent agreement with the pattern of the first PC score. Its temperature anomaly range is of the same order as the direct composite field. A good example of inverse correlation between the PC score and the real field is that of September 1985 (not shown).

The PC loading time series does not show a distinct periodicity that could point to a seasonal behaviour in the occurrence of the spatial pattern described by the PC score. The calculation of the spectrum yields only one peak, greater than the $90 \%$ confidence level, with a 2.4-month period. Another spectrum calculation in which all the PC loading values within \pm 0.3 were set to 0 yielded practically the same result, also with only one significant peak with the same periodicity. The calculated linear trend does not yield a well-determined slope, and hence the frequency distribution of this mode does not appear to be undergoing any modifications in time. 


\section{2. $P C 2$}

The PC score for the second PC (Figure 3(a)) shows a quasi-circular negative anomaly pattern over Antarctica, surrounded by a weak warm anomaly ring, which extends into the tropics. The most negative values of the Antarctic region are not centred over the continent, but are located over Wilkes Land. The anomaly over Antarctica is far more significant than that found at mid-latitudes, i.e. approximately seven times the mid-latitude anomaly in the direct mode. This mode represents $19.3 \%$ of the total variance. In its direct form, i.e. as described above, the pattern corresponds to $8.48 \%$ of the total variance.

The direct mode anomaly field, with a cooler Antarctica and somewhat warmer mid and low latitudes leads to an increase in the temperature gradients between the tropics and the polar region, in winter and a weakening of the gradient in summer. This implies a strengthening of the westerly flow in winter and consequently of the polar vortex and a weakening of the easterly flow in summer. The most relevant examples for this PC can be found for September, October and November 1987, coincident with the El Niño event discussed in Part I. This period corresponds to the first very deep ozone 'hole' (see references in Part I) recorded over Antarctica since 1985. This too was the first ozone 'hole' to cover an area larger than Antarctica. The composite real field (Figure 3(b)) is in excellent agreement with the PC score pattern, though it appears to over a slightly larger area of the polar and sub-polar regions with a negative temperature anomaly. This negative anomaly reaches a value of $-2.1 \mathrm{~K}$ over Wilkes Land.

The inverse mode, with a warm anomaly over Antarctica and cooling in temperate regions also occurs (Figure 3(c)). As was the case for the direct composite map, the polar region anomaly extends, this time

a)

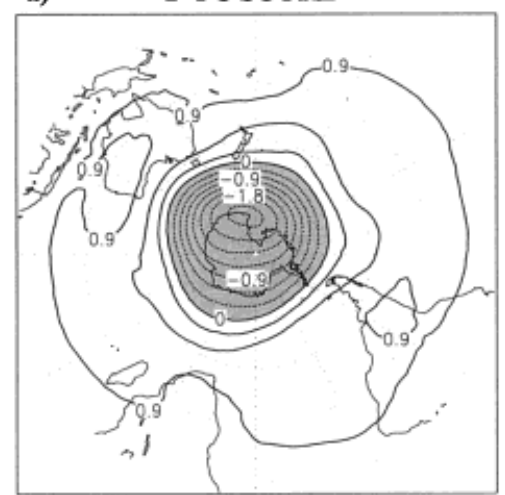

b)

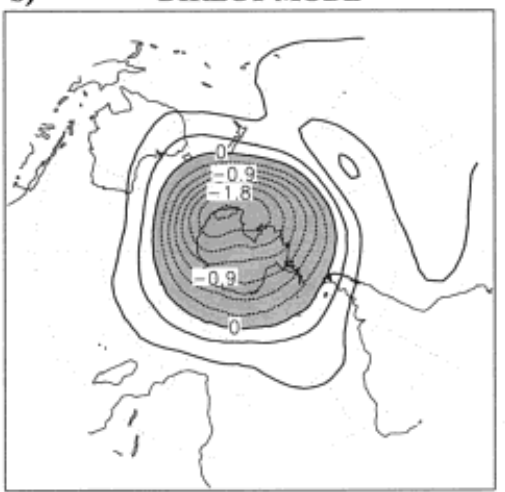

c) INVERSE MODE

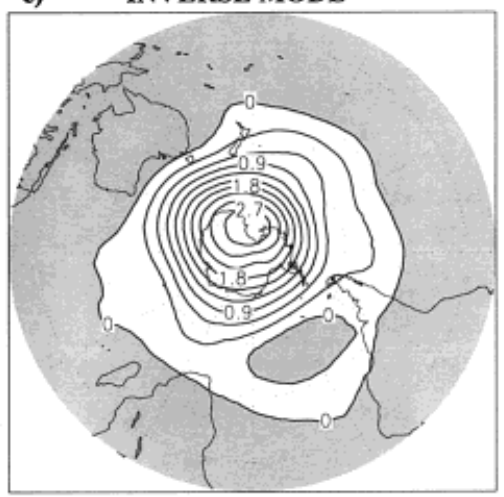

e) PC LOADING SPECTRUM
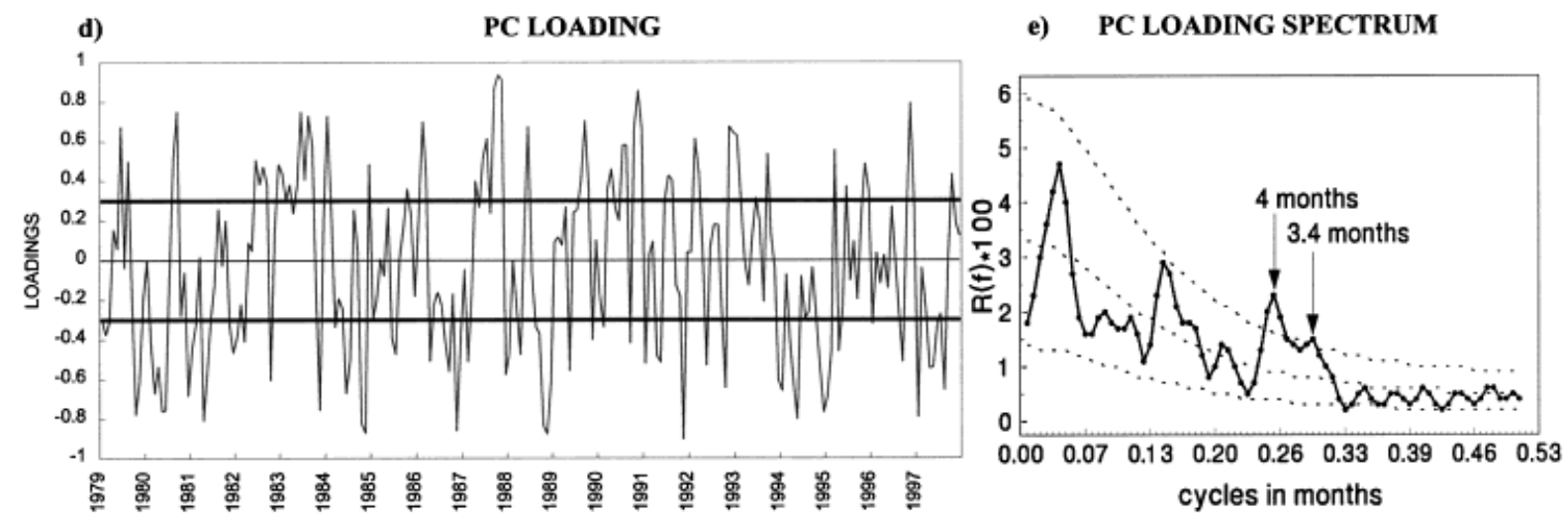

Figure 3. Main aspects of the second PC: (a) PC score spatial pattern, contour values are every 0.5 with shaded areas for negative score values; (b) real field composite for direct mode events, in K; (c) real field composite for inverse mode events, in K; (d) PC loading time series and the hyperplane levels for \pm 0.3 ; (e) normalized spectral analysis according to Tukey with a Parzen window given as $R(f) \times 100$ versus cycles/month (full line) and the $90 \%$ confidence level considering theoretical Markov process. Significant peaks are highlighted 
with a positive value, into sub-polar regions and even gets close to Australia, New Zealand, Chile and Argentina. The positive range is $2.7 \mathrm{~K}$. As a matter of fact this mode is somewhat more frequent than the direct mode since it describes $10.84 \%$ of the total variance. A look at the PC loading time series (Figure 3(d)) shows that this is effectively the case. This pattern mitigates the tropical/polar temperature gradient in winter and strengthens it in summer. Hence, its occurrence implies a weakening of the westerly flow in winter and a strengthening of the easterly flow in summer. The most significant inverse mode example can be found in November 1991, when the correlation between the inverse PC and the data is -0.9 . It is interesting to note, however, that a similar behaviour is also found in October and November 1988 when the PC loading reached values of -0.83 and -0.88 , respectively. As mentioned in Part I, the ozone 'hole' practically did not exist during the Austral spring of 1988. The fact is that there is a significant contribution to the anomaly field by this PC during 1987 (El Niño) and 1988 (La Niña) in direct and inverse modes, respectively, which are associated with extreme behaviours in the state of the ozone layer there. This could appear to imply that this mode is the consequence of dynamic processes rather the response to long-term changes to the ozone layer. There does not appear to be a direct relationship between the occurrence and significance of this pattern and the depth and extent of the ozone 'hole' during the 1990s. Thus, ozone depletion would not necessarily be the primary cause of this behaviour. Since at this time it is not possible to discuss further the possible link between El Niño/La Niña years because of the consequences of volcanic activity masking other ENSO examples, as well as decadal variability, it is not possible either to understand fully the role of dynamics on the ozone levels.

The spectral analysis (Figure 3(e)) shows the existence of a cycle of approximately 4 months in the occurrence of this mode, together with a less significant one of about 7 months. There also appears a peak under the $90 \%$ confidence level, which represents a 25.3 -month period. However, due to its low significance and the behaviour observed in the PC loading series, it would appear that this refers to the modulation of the recurrence of the spatial pattern.

\section{3. $P C 3$}

The third PC (Figure 4(a)) yields a pattern that is similar to that of PC 1, but rotated $90^{\circ}$ with respect to that mode. This mode has an explained variance of $13.8 \%$, which is a fairly significant reduction with respect to the first two PCs. The direct pattern, shown in Figure 4(a), describes $7.61 \%$ of the total variance. It presents a negative anomaly over the Weddell Sea that extends into the South Atlantic mid-latitudes and Patagonia, while the positive anomaly is located on the other side of Antarctica and adjacent seas. The observed direct mode composite field can be seen in Figure 4(b). As in the previous cases, the agreement between the PC score map and the composite field is very good. The dipole structure has temperatures between 1.5 and $-1.5 \mathrm{~K}$.

Inspection of the PC loading time series variability shows that after 1990 this mode becomes far more significant. Before that, the positive correlation values barely reached 0.6. Yet after 1990 there are 13 events where the correlation value exceeds 0.6 (Figure 4(d)). This implies an important change in the occurrence of this particular mode after 1990. As a matter of fact the most prominent case occurs in December 1996, when the PC loading values is 0.9 . This result is effectively pointing to a more frequent cool anomaly over the Weddell Sea region, which is only partially compensated by a warming on the other side of Antarctica. Due to such an increase in the amplitude of the PC loading, this pattern becomes more representative of the anomaly fields observed in the region during the Austral spring and the beginning of summer during the second half of the sample.

It is interesting to note that during October 1980, 1983 and 1984 the monthly mean total ozone perturbations (Wirth, 1991) have a pattern that is close to that observed here for the lower stratospheric temperature anomaly. While the PC loading values at these times are not as extreme as for the examples mentioned above, they are always greater than 0.3 .

The inverse mode has a positive anomaly over the Weddell Sea, a negative one south of New Zealand and it explains a somewhat smaller variance, i.e. $6.22 \%$ of the total variance. Such a relatively low explained variance be clearly seen in the PC loading time series, where there are only nine events when 
a)

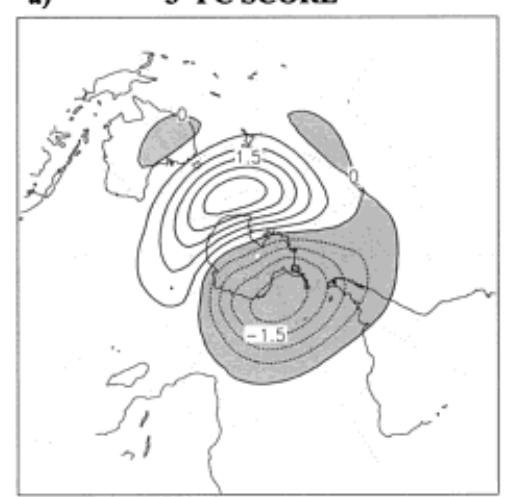

b)

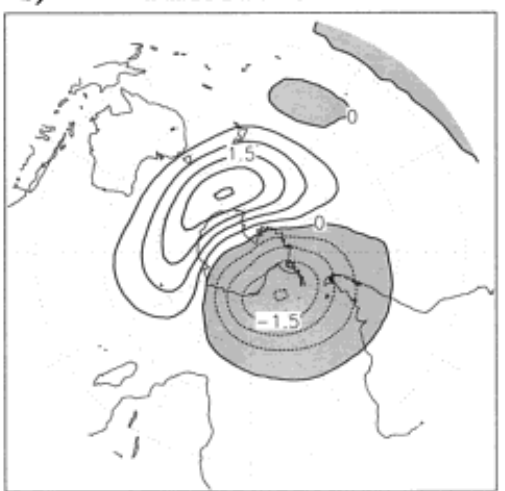

c)

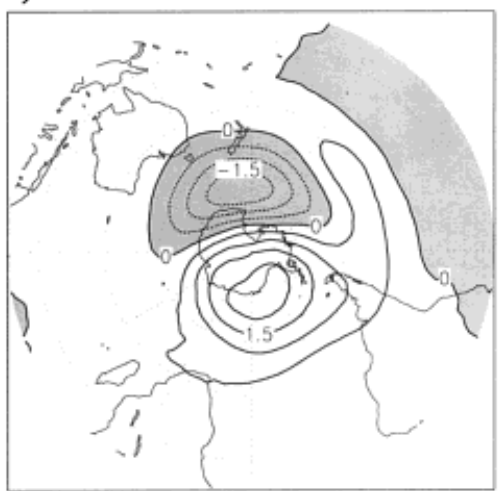

d)

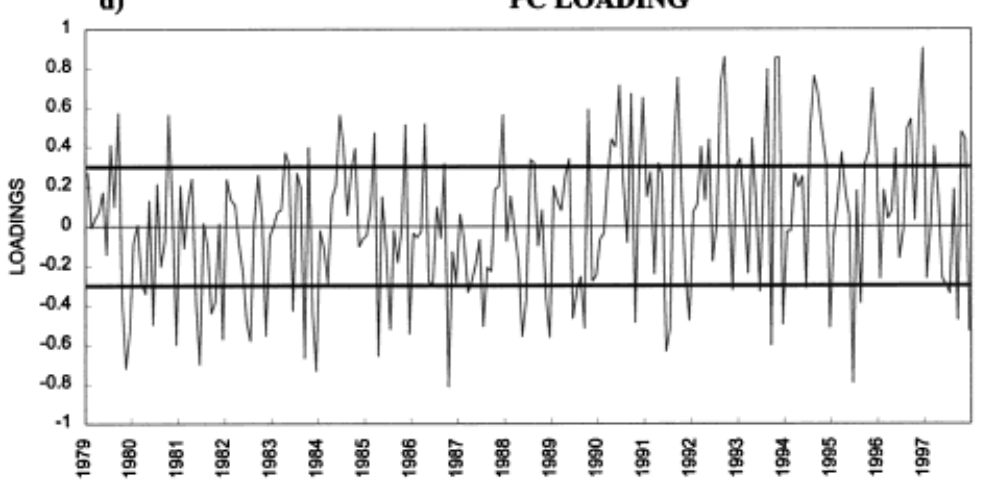

Figure 4. Main aspects of the third PC: (a) PC score spatial pattern, contour values are every 0.5 with shaded areas for negative score values; (b) real field composite for direct mode events, in K; (c) real field composite for inverse mode events, in K; (d) PC loading time series and the hyperplane levels for \pm 0.3

there is a good negative correlation i.e. less than -0.6. Furthermore, they are more or less homogeneously distributed throughout the sample period and thus its significance does not change during the sampled period. The inverse mode composite field can be seen in Figure 4(c), also showing a good agreement between the PC score and observations. It must be noted that the composite inverse mode appears to be slightly rotated towards the east with respect to the PC score and the direct composite mode. Nevertheless, the range of the temperature perturbation remains the same. A good example of this behaviour took place in October 1986. In this case the lower stratosphere over the Weddell Sea was approximately $5^{\circ} \mathrm{C}$ warmer than the average. At this time there is no equivalent calculation of the total ozone perturbation field for comparison with the temperature behaviour.

This is the first PC that has a positive trend in the PC loading time series, as would be expected from the enhancement in the occurrence of the direct mode after 1990 and the more or less homogeneous occurrence of the inverse mode.

\section{4. $P C 4$}

The amount of variance this mode explains again shows a significant decrease with respect to previous ones. The fourth PC (Figure 5(a)) only represents $7.5 \%$ of the total variance. In its direct mode it has a distinct annular dipole structure, with a warm core over central Antarctica and cool area between 75 and $30^{\circ} \mathrm{S}$. The pattern also has an outer ring of positive anomalies extending towards the Equator. This outer ring shows three regions of enhanced anomaly amplitude, with a quasi-wave 3 spatial distribution. The direct mode represents $4.51 \%$ of the total variance, while the inverse mode explains somewhat less 
a)

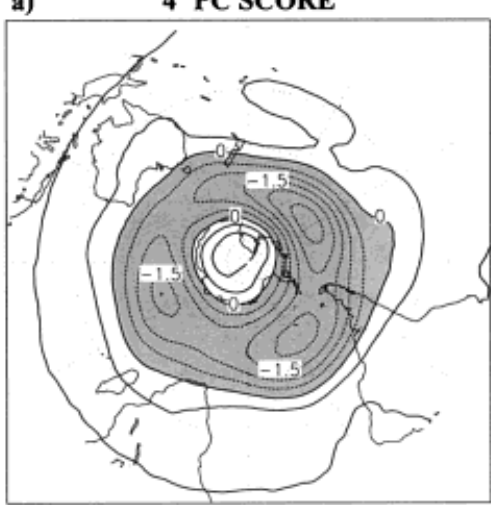

b)

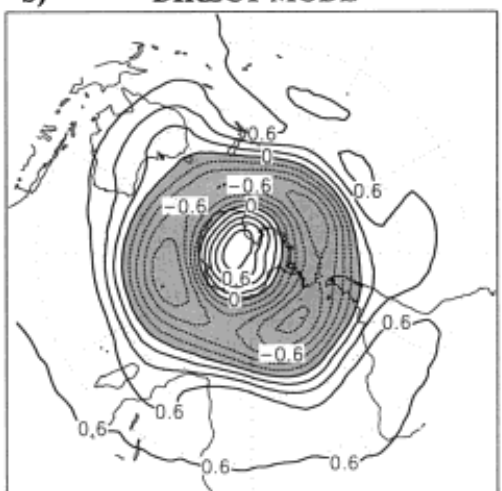

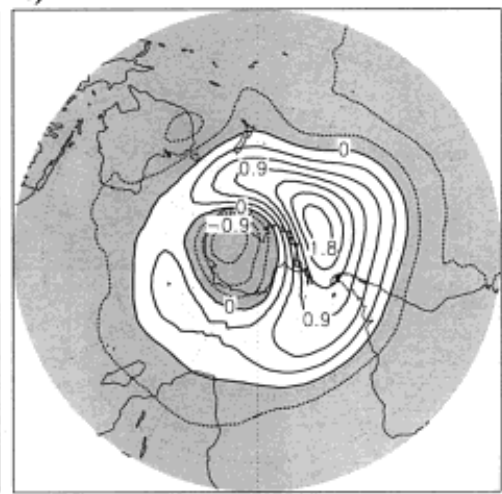

d)

PC LOADING

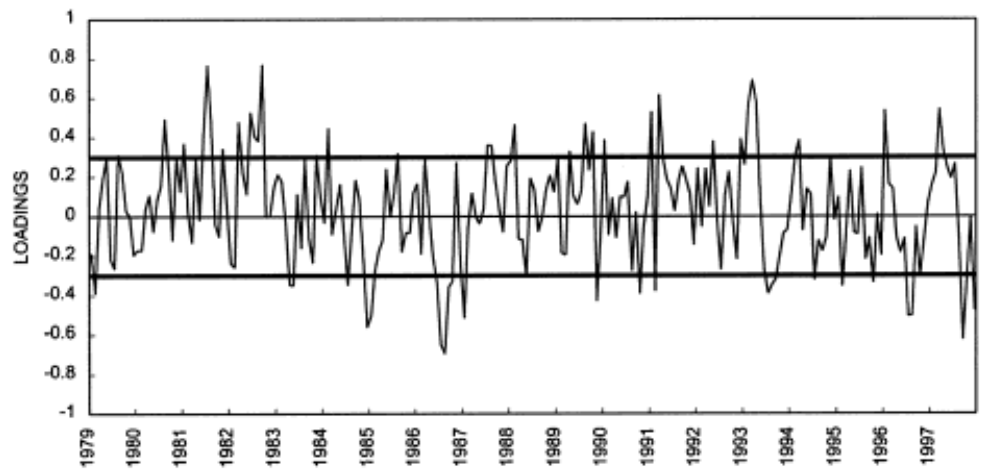

Figure 5. Main aspects of the fourth PC: (a) PC score spatial pattern, contour values are every 0.5 with shaded areas for negative score values; (b) real field composite for direct mode events, in K. (c) real field composite for inverse mode events, in K; (d) PC loading time series and the hyperplane levels for \pm 0.3

variance: 3.01\%. The composite observed field, shown in Figure 5(b), has a spatial structure in good agreement with the PC score pattern, including the wave-3 distribution at mid-latitudes. The range of the temperature anomaly is weaker than for the previous PCs. The inverse mode, shown as the composite real field in Figure 5(c), corresponds to a cool area over Antarctica and the tropics, with a warm ring in between. However, the relationship between this composite field and the corresponding PC score pattern is not as good as in all of the above cases. While the general features are preserved, they are not located more or less exactly over the same geographical features as was the case of the other examples. Furthermore, the wave-3 distribution at mid-latitudes is no longer there. The warm outer ring shows a temperature anomaly that, in absolute values, is twice that of the Antarctic core.

The inspection of the corresponding PC loading time series (Figure 5(d)) shows that the direct mode virtually vanishes between 1983 and 1990 since almost all the positive correlation values fall within the hyperplane. The direct mode is more representative of the behaviour of the temperature anomaly fields between 1980 and 1983, as well as between 1990 and 1994. An example of a good fit to a real anomaly field by this pattern is found in September 1982 (not shown). The inverse mode has a lower frequency of occurrence, as can be seen in Figure 5(d). Most of the temperature anomaly fields described by this mode occurred between 1983 and 1990. An example of such a field can be found in July 1986. There are two more events with a similar level of significance.

In general, the PC loading time series shows high frequency variability modulated by what would appear to be a quasi-10-year signal. By modulating oscillation we mean that the direct mode prevails for a 5-year period and is subsequently replaced by a 5-year span where the inverse mode prevails. However, as is mentioned in previous examples in Part I, the PC loadings time series is too short to corroborate the existence of decadal variability. 


\section{5. $P C 5$}

The fifth PC (Figure 6(a)) explains $4.9 \%$ of the total variance, $3.1 \%$ corresponding to the direct mode, and $1.8 \%$ to the inverse mode. This means that the direct mode, within the limitations of such a low total variance, can be observed in the temperature anomaly fields much more frequently than the inverse mode. Inspection of the PC loadings time series (Figure 6(d)) shows that despite this comparatively low variance there are a fairly large number of occurrences outside the hyperplane, both in direct and inverse modes. The spatial structure of this mode is more complex than in previous cases. The PC score pattern has a quasi-wave 2 structure of negative anomalies at mid and high latitudes, surrounded by a ring of positive anomalies at $30^{\circ} \mathrm{S}$ of the Equator, which appear to have a weak wave $3 / 4$ structure, and another ring of negative anomalies in the tropics. The negative anomaly centres at mid to high latitudes are located over Patagonia and the South Pacific, separated by weakly positive temperature values. The equatorial/tropical ring is not circular and has a fairly significant southward displacement into the subtropics over the Central Pacific.

Inspection of the direct and inverse composite maps shows in the first place that the direct composite map is closer to the PC score spatial distribution than the inverse mode composite map. This is reasonable given that the direct mode almost explains two thirds of the variance associated with this PC. The direct mode composite map (Figure 6(b)) shows a slightly narrower equatorial anomaly region, particularly over Indonesia and northern South America. This implies that its southward projection over the Central Pacific Ocean is more distinct. The mid-latitude region is a bit more complex than for the PC score pattern, while the high latitude region has a similar spatial pattern to that of the PC score, albeit slightly rotated towards the west. The inverse mode composite (Figure 6(c)) yields a slightly wider equatorial

a)

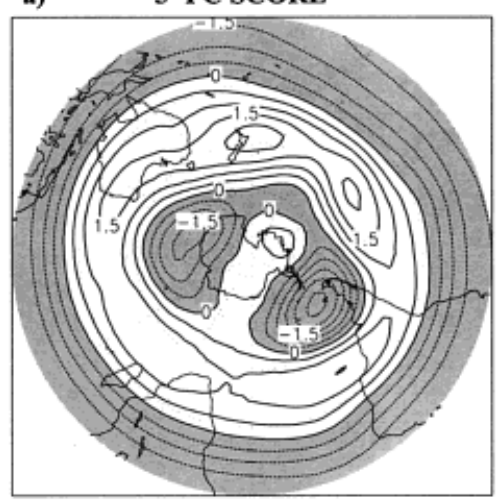

b)

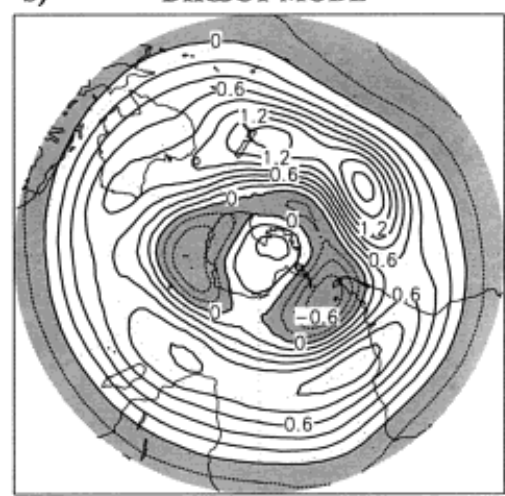

c) INVERSE MODE

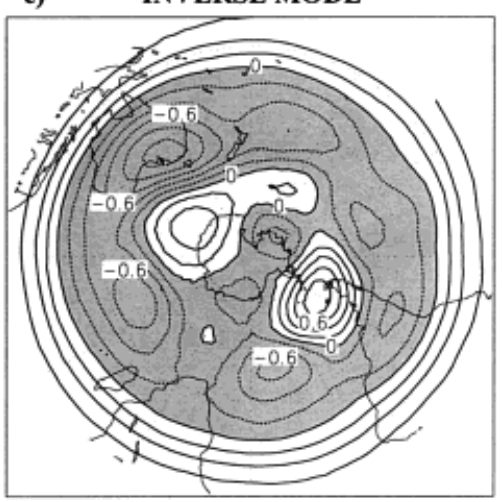

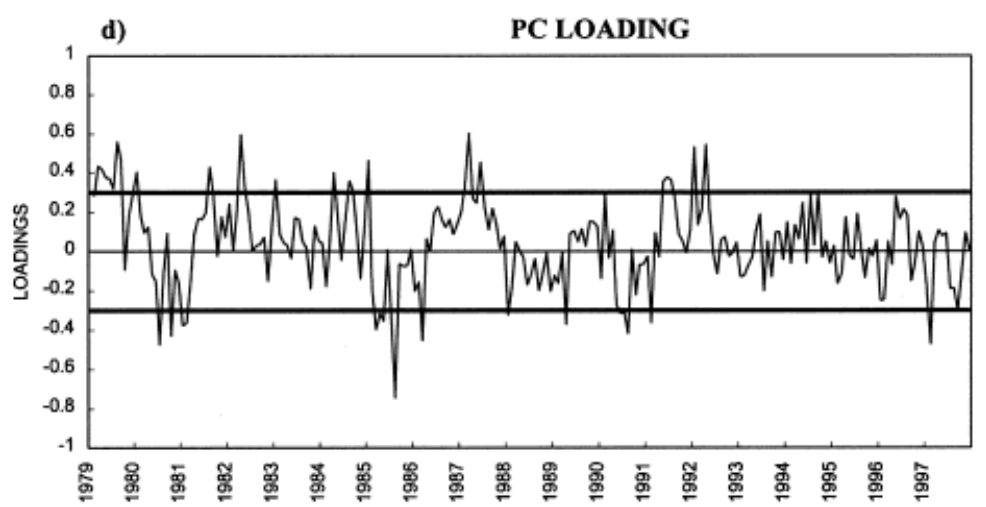

Figure 6. Main aspects of the fifth PC: (a) PC score spatial pattern, contour values are every 0.5 with shaded areas for negative score values; (b) example of the direct mode: spatial anomaly temperature at $70 \mathrm{hPa}$ for March 1987; (c) example of the inverse mode: spatial anomaly temperature at $70 \mathrm{hPa}$ for August 1985; (d) PC loading time series and the hyperplane levels for \pm 0.3 
anomaly region, which has similar, though not as prominent features, to the PC score and the direct mode. The mid-latitude region preserves the ring-like spatial distribution with changes in the finer structure, while the high latitude region breaks up into two isolated core areas. A good example of an anomaly field fitted by this pattern corresponds to March 1987, while a good example of the inverse mode can be observed in August 1985.

The PC loading time series (Figure 6(d)) has a number of features of interest. Firstly, the occurrence of such spatial patterns, both in direct and inverse state started to lose their significance after 1992. In particular, during this latter period there are only three cases in which the correlation values do not belong to the hyperplane. It must be pointed out that even before 1992 the PC loading values are also rather low. Secondly, the behaviour of the PC loading time series shows an interesting clustering of direct or inverse mode anomalies before 1992. This behaviour points to the existence of low or mid-frequency cycles in the occurrence of this mode, which were not observed in the previous modes. The clustering of direct or inverse events would appear to be related to the phase of the QBO. Inspection of Figure 6(d) and the comparison with the QBO phase at $70 \mathrm{hPa}$, shown in Figure 6 of Part I suggests that the occurrence of this mode is related to the change in phase of the QBO at $70 \mathrm{hPa}$ until 1992. Indeed the inverse mode coincides with east to west phase transitions during 1980, 1985 and with a weaker correlation during 1983 and 1991. The direct mode appears during the west to east transitions in 1982, 1984, 1987 and 1992. This pattern would suggest a possible QBO-related temperature field extending into the subtropics with an anti-phase at mid-latitudes, which used to occur, at least within the present data set, only during certain stages of the QBO development. Up until 1992 this field pattern appears only during the transition from one QBO phase to the next, while other field patterns are dominant the rest of the time. As always, high frequency variance is also present. Finally, it is interesting to note that if this spatial distribution is associated with the QBO evolution, its equatorial structure implies that the associated temperature anomaly is not purely zonal that there is a deviation from such behaviour, particularly over the Pacific Ocean.

\subsection{Summing up}

Before proceeding further, it must be noted that the Lev diagram (Figure 1) shows a feature regarding PC 1 and PC 2 that needs to be considered. The second PC's explained variance is very similar to that of the first PC (19.3\% and 20\%, respectively). This is due to the fact their eigenvalues are very close. According to North's Test (North et al., 1982), the structures observed for PC 1 and PC 2 could be 'degenerate' and as such not be representative of the real patterns in the observed fields. Due to this, it is generally recommended that, when such a case arises, a rotation of the PC components be carried out, given that the rotation process is an attempt to improve the interpretability of the solutions. In our particular case the patterns obtained for the PC 1 and PC 2 resulted in very good and even excellent representations of the real anomaly fields. Furthermore, the excellent agreement between the composite fields associated with the direct and inverse modes and the corresponding PC scores patterns show that in this particular case the modes obtained are good representations of physical realizations. Hence, it was not necessary to carry out the rotation in order to determine or infer physical properties for these patterns.

Because the PC score patterns obtained in the present study refer to real anomaly fields, it is convenient to describe them in terms of zonal stationary or quasi-stationary planetary wave-like structures. The first PC modes have simple structures, which can be described in terms of basically a one wave pattern, while the higher order ones require more than a one wave pattern for their full description. Thus, in terms of waves the T-mode patterns are:

(i) Quasi wave 0: second PC. The Antarctic and surrounding oceans region is covered by a deep anomaly field. This mode does not show a trend or enhancement in the frequency of occurrence either of the direct or inverse modes. It appears to be influenced by ENSO, but the sample considered is too short to obtain valid conclusions regarding this. 
(ii) Quasi-wave 1: first and third PCs. These two wave 1 patterns are at right angles to each other and cover mid to high southern latitudes. There is no change in the behaviour of the first PC throughout the sample, nor does it present a distinct season behaviour, or preferred time of occurrence. The third PC has a well-defined trend with an enhancement in the occurrence of the direct mode, i.e. cooling over Patagonia, during the 1990s,

(iii) Combined wave 0 with wave 3: fourth PC. Two regions are observed in this pattern, a core centred over Antarctica and an outer ring at southern mid to subtropical latitudes with a quasi wave 3 structure. This PC appears to alternate between a predominance of the direct and inverse mode with an apparent quasi-decadal modulation. Hence, it contributed to an enhancement of the stratosphere over Antarctica between 1983 and 1990.

(iv) Combined wave 0/1, 2 and waves 3/4: fifth PC. This mode is defined by an outer wave $0 / 1$ structure over the Equator, with a quasi wave 3/4 anomaly of opposite sign over the subtropics and a wave 2 pattern at mid to high latitudes, including Antarctica. This PC appears to be linked to the QBO, specifically appearing during the change in phase of the equatorial wind. However, it loses significance after 1990 and hence it is difficult to relate the weakening of this spatial pattern to the behaviour of the QBO during the period.

\section{FINAL DISCUSSION OF THE S- AND T-MODE RESULTS}

In general, and as can be particularly observed in the results of the T-mode analysis, the eigenvalue, and the amount of variance explained, is proportional to the number of original variables in the sample explained by a particular model given by the PC scores. For example, the explained variances for the fifth PC, described above, are $3.07 \%$ for the direct mode and $1.85 \%$ for the inverse mode. The number of occurrences, when the PC loading time series values do not belong to the hyperplane is 25 and 15 , respectively. The total explained variance has approximately the same ratio as the number of occurrences. Similarly, in the case of the S-mode (Part I) the variance explained by the PC score time series is proportional to the number of grid points included in the areas where the PC loadings also lay within the hyperplane.

This rather direct relationship between the explained variance and the number of events or occurrences in the case of the T-mode and the number of grid points in a cluster in the S-mode approach has a rather straightforward explanation. It is due to the fact that in all these cases the types obtained tend to correspond with simple, distinct structures in the observations.

It is for this reason that the S-mode PC 2 pattern, which has an area of influence over Antarctica, comes second after the tropical/subtropical mode. Indeed at high latitudes there is a concentration of grid points since the database used is built in a fixed latitude-longitude format. If an analysis that described the behaviour of the relevant S-mode PC loadings in terms of the area were necessary, a method would be required that transformed the latitude-longitude grid point data set into an equal area grid (as for example in Richman and Gong, 1999). This change would modify the amount of explained variance and change the order of the PC models, especially in the S-mode, but not necessarily the patterns of the PCs. The T-mode results could suffer in some cases minor changes in the PC variance and other results (Compagnucci and Ruiz, 1992).

The inspection of the variance tables included in Figure 1, both for the S-mode (Part I) and T-mode of the MSU data set considered here shows different variance distributions. This is clear evidence that these two approaches yield different results, as discussed above. The S-mode has at least six independent eigenvalues, which can represent as many orthogonal time patterns or series. The T-mode only yields five PCs significantly different from noise, which represent the five leading spatial patterns for the temperature anomaly fields that may occur in direct or inverse modes.

Both methods yield results with different physical meanings that are complementary and mutually exclusive. The S-mode, as discussed in Part I, is useful for the temporal analysis of the variable under 
study, clustering the results in areas where the temporal behaviour at the individual grid points is similar or close. The T-mode, discussed above, describes the main spatial patterns of the variables considered, determining when the patterns obtained can describe the field under study.

A quick inspection of the PCs for the S- and T-mode approaches may lead to a prompt conclusion that the results are very similar. This is specially so if these conclusions are drawn by inspecting the S-mode PC loading maps, which are correlation maps, and the T-mode PC score maps, which are normalized representations or types of real anomaly fields, as shown by the composite anomaly maps for times during which they do not belong to the hyperplane. In particular, it would be tempting to think that the following results are at least similar or equivalent:

(i) T-mode PC 1 and S-mode PC 3, i.e. the South Pacific-South Indian Ocean dipole;

(ii) T-mode PC 2 and S-mode PC 2, i.e. over the Antarctic region;

(iii) T-mode PC 2 and S-mode PC 1, mainly over Antarctica

(iv) T-mode PC 3 and S-mode PC 4, i.e. the cross dipole over the Weddell Peninsula and south of Australasia;

(v) T-mode PC 5 and S-mode PC 5, particularly in the equatorial region.

Note that in this comparison the tropical/subtropical region represented by the S-mode PC 1 does not appear to have an equivalent T-mode. Nor does there appear to be an equivalent S-mode for the T-mode PC 4.

If such similarity or equivalence relationships were to exist between the S- and T-mode results, it would imply that simple, linear physical relationships between space and time could be found. Then a fairly straightforward function would transform spatial variance into temporal variance.

Yet a more profound analysis of these potential space-time relationships requires as well the consideration of the PC score time series patterns obtained in the S-mode approach and the PC loadings correlation time series of the T-mode results. These two sets of results must necessarily be considered together.

In order to test objectively the similarity of the S- and T-mode results, the following approach was followed. Given that in the S-mode approach the standard deviation (SD) value of each grid point time series is removed during its normalization, and given that in the T-mode approach the SD value of each spatial field is removed for the same purpose, the appropriate comparison must be made in a different manner. Comparable fields and time series were obtained by:

(i) multiplying the S-mode PC loadings maps at each grid point, by the corresponding SD value obtained from the grid point time series;

(ii) multiplying the T-mode PC loading time series at each time by the corresponding SD value obtained from each real spatial field.

These new spatial and temporal patterns, respectively, are no longer normalized by the SD and can thus be adequately compared with the T-mode PC score maps and the S-mode PC score time series, respectively. If both comparisons, i.e. the spatial and temporal comparisons, now yield similarities or equivalent patterns, then there does exist a single, simple, linear space-time relationship, i.e. there is a dominant forcing mechanism linking space and time. However, if the comparison of either the spatial or temporal pattern falls then there is no such relationship.

The possible relations mentioned above were thus further analysed using this approach, calculating the correlation of the temporal patterns and the spatial patterns, respectively, derived as above. While the resulting spatial and temporal patterns are not shown, the corresponding correlation tables are presented in Tables I and II. The results show that two of these relationships appear to have a linear physical process that strongly link the results of the $\mathrm{S}$ - and T-mode analysis.

The highest correlation obtained, above 0.9, corresponds to the T-mode PC 1 and the S-mode PC 3 ( -0.9471 and 0.9068$)$. The dipole anomaly field pattern and its occurrence, found with the T-mode, is thus strongly linked to the S-mode PC 3 time series which occur in approximately the same areas of the hemisphere. Such a close relationship means that the field, observed in the T-mode PC 1, is the simple 
Table I. Correlation between the PC loadings (T-mode) multiplied by the S.D. of each field and the PC scores in S-mode

\begin{tabular}{|c|c|c|c|c|c|c|}
\hline & $\begin{array}{l}\text { 1st PC } \\
\text { score }\end{array}$ & $\begin{array}{l}\text { 2nd PC } \\
\text { score }\end{array}$ & $\begin{array}{l}\text { 3rd PC } \\
\text { score }\end{array}$ & $\begin{array}{l}\text { 4th PC } \\
\text { score }\end{array}$ & $\begin{array}{l}5 \text { th } \mathrm{PC} \\
\text { score }\end{array}$ & $\begin{array}{l}\text { 6th PC } \\
\text { score }\end{array}$ \\
\hline$(1$ st Loading $) \times \sigma$ & -0.1687 & 0.2125 & -0.9471 & -0.0438 & 0.1163 & -0.0172 \\
\hline$(2$ nd Loading $) \times \sigma$ & 0.4435 & -0.8236 & -0.2713 & 0.1977 & -0.0818 & -0.0501 \\
\hline (3rd Loading) $\times \sigma$ & -0.0163 & -0.3592 & -0.0336 & -0.9121 & -0.0517 & 0.1127 \\
\hline$(4$ th Loading $) \times \sigma$ & 0.3187 & 0.2301 & -0.0440 & -0.1390 & 0.4888 & 0.7175 \\
\hline$(5$ th Loading $) \times \sigma$ & 0.1778 & -0.0452 & 0.0998 & -0.1122 & -0.7348 & 0.4699 \\
\hline
\end{tabular}

Table II. Correlation between the PC loadings (S-mode) multiplied by the S.D. of each grid point and the T-mode PC scores

\begin{tabular}{lccccrc}
\hline & $\begin{array}{l}\text { (1st Load- } \\
\text { ing }) \times \sigma\end{array}$ & $\begin{array}{l}\text { (2nd Load- } \\
\text { ing }) \times \sigma\end{array}$ & $\begin{array}{l}\text { (3rd Load- } \\
\text { ing }) \times \sigma\end{array}$ & $\begin{array}{l}\text { (4th Load- } \\
\text { ing }) \times \sigma\end{array}$ & $\begin{array}{l}\text { (5th Load- } \\
\text { ing }) \times \sigma\end{array}$ & $\begin{array}{l}\text { (6th Load- } \\
\text { ing }) \times \sigma\end{array}$ \\
\hline 1st PC score & -0.2657 & -0.2353 & 0.9068 & -0.0479 & -0.1214 & -0.0181 \\
2nd PC score & 0.8362 & 0.8802 & 0.2876 & 0.2162 & 0.1267 & -0.1408 \\
3rd PC score & 0.1063 & 0.3395 & 0.0395 & -0.9096 & 0.0541 & -0.1496 \\
4th PC score & 0.4298 & -0.1100 & -0.0153 & -0.1464 & -0.4738 & 0.7211 \\
5th PC score & -0.0473 & -0.0574 & 0.0746 & -0.1079 & 0.7685 & 0.4579 \\
\hline
\end{tabular}

linear response in space to a dominant forcing that resulted in the temporal behaviour of temperature described by the S-mode PC 3. In other words, there is a simple dominant physical mechanism that forces the temperature anomaly over the Southern Hemisphere and results in an anomaly field pattern for all of it. Similar, if somewhat slightly smaller correlation values were obtained for the comparison of the T-mode PC 3 with the S-mode PC 4 ( -0.9121 and -0.9096$)$.

The next best correlation is found between the T-mode PC 2 and S-mode PC 2. The correlation values obtained for both comparisons $(-0.8236$ and 0.8802$)$ show that this relationship is somewhat weaker than the previous ones but still relevant. This result also implies that the spatial anomaly field over high southern latitudes, and its sign, is determined by the behaviour of the time series corresponding to the grid points over Antarctica. Finally, there is a somewhat weaker correlation $(-0.7348$ and 0.7685$)$ between both PC 5, which referred to the QBO evolution in space and time. This result implies that there is indeed a relation between the QBO phase and the spatial temperature anomaly response.

The correlations between the remaining $\mathrm{S}$ - and T-mode leading components are low and thus not significantly different from zero. While these components independently exist either in space or time, there is no simple linear mechanism that can link the results of both approaches. For example, the volcanic signatures found in the S-mode PC 1 time series, despite their prominence, do not appear to have a direct impact on the spatial distribution of the temperature anomaly fields. Nor does there appear to be a relationship between the S-mode PC 1 and the T-mode PC 2 despite their apparent similarities over Antarctica.

The above discussion points to the importance of processing the data using both the T- and the S-mode approaches in order to reach useful physical insights. The existence of possible physical processes linking $\mathrm{S}$ - and T-mode results does not imply by any means that the T- and S-mode approaches are statistically linked with one another: the results of each mode indeed remain statistically complementary. While the T-mode analysis shows that, for example, the negative anomaly pattern over Antarctica becomes more common, it does not tell us anything about the cooling trend itself. The S-mode needs to be used to infer the regional trend behaviour there but it does not tell us the spatial pattern of the field.

The analysis of the main spatial patterns for the lower stratospheric anomaly temperature fields, using the T-mode approach, show that the two main spatial types, given by the first and second PC score, do not undergo changes in their frequency of occurrence during the period under study. This then implies 
that the significant trends observed in the S-mode approach are probably not significantly influencing the patterns of these spatial distributions over the Southern Hemisphere observed in the T-mode analysis. The higher order T-mode PCs $(\geq 3)$ are the ones that show changes in their frequency of occurrence and their significance before and after 1989-1990. In particular, the fifth PC loses its significance during the latter part of the sample. This means that the amount of variance it can describe for a given field or 'snapshot' decreases, and furthermore this variance tends to diminish with time to hyperplane values. Thus, this model becomes irrelevant to describe the observed fields. The T-mode fourth PC loading time series would appear to have a quasi-decadal modulation, with significant alternation between the direct and inverse modes. However, a longer time series is needed to validate this long-term behaviour. The third mode shows a different behaviour. In this case the direct mode, with a deep negative anomaly field over Patagonia and Antarctica, becomes far more relevant and frequent during the second half of the sample, while the inverse mode shows no changes. Hence the changes in the frequency distribution of the T-mode third, fourth and fifth PC may represent modifications in physical processes that affect the behaviour of the temperature anomaly fields in the Southern Hemisphere.

The S-mode results show that most areas have negative temperature anomaly trends, and in particular the Antarctic region has the most negative trend, while the region over and south of Oceania presents no significant trend during the period under study. Regarding the latter, the good relationship between the T-mode PC 3 and the S-mode PC 4, observed above, the fact that the negative anomaly over Patagonia and the Weddell Sea is becoming more common, while little or no change is observed at the other branch of the dipole is an interesting point. This implies that there is a regional forcing mechanism that is becoming more significant on one side of Antarctica and not the other.

The results presented above, with changes in temporal trends (S-mode) and changes in the relevance of particular anomaly patterns for the temperature anomalies (T-mode) over the Southern Hemisphere, do seem to point to the consequences of ozone depletion at high southern latitudes. Others appear to identify possible stratospheric cooling resulting from ozone depletion and possibly greenhouse gas accumulation and tropospheric warming as described in Ramaswamy et al. (1996) and Tett et al. (1996). The S-mode trend results point to the possible combined effect of ozone depletion and greenhouse warming in agreement with Graf et al. (1998).

Nevertheless, it is not easy to pinpoint the causes that lead to changes in the occurrence of the different spatial patterns (T-mode) discussed above. Such changes imply modifications in circulation patterns. There is a weak evidence of apparent quasidecadal or long-term cycles influencing the distribution of the temperature anomaly field. Furthermore, despite the limited time span considered in this study, there appears to be a significant change in the behaviour of the temperature anomaly between the late 1980s and early 1990s. It is not possible at this stage to determine the physical processes that are leading, for example, to an enhanced occurrence of the third PC direct mode (T-mode). Is the cooling trend observed over Patagonia and the South Atlantic the result of the ozone depletion, or is depleted ozone being transported there because of circulation changes, enhancing the cooling there, for example? This process must be significant since as discussed above there is a coupling between the independent results from the two analysis techniques. Further studies are necessary in order to determine the links between the present results and the atmospheric processes involved.

\section{ACKNOWLEDGEMENTS}

The research was supported by InterAmerican Institute for Global Change research IAI-ISP-111-076 project, by the Agencia Nacional Para la Promoción de la Ciencia y la Tecnología (ANPCYT-Argentina) PICT-97-02197 project and the Consejo Nacional de Ciencia y Técnica (CONICET-Argentina) PIP No. 0428/98 project and UBA TW06/98-2000. The authors are indebted to Rolando Garcia and Mike Richman for their comments and suggestions. The authors also wish to thank the Marshall Space Flight Center for the access to the MSU data, as well as Dr. David Karoly and an anonymous reviewer that contributed with valuable comments and suggestions. 


\section{REFERENCES}

Angell JK. 1988. Variations and trends in tropospheric and stratospheric global temperatures, 1958-87. Journal of Climate 1: $1296-1313$.

Cattell RB. 1952. Factor Analysis. Harper and Row: New York.

Compagnucci RH, Salles MA. 1997. Surface pressure patterns during the year over southern South America. International Journal of Climatology 17: 635-653.

Compagnucci RH, Vargas WM. 1998. Inter-annual variability of the Cuyo Rivers' streamflow in the Argentinean Andean mountains and ENSO events. International Journal of Climatology 18: 1593-1609.

Compagnucci RH, Ruiz N. 1992. On the interpretation of the Principal Component Analysis as applied to meterological data. In 5th International Conference on Statistical Climatology, Toronto, Canada, Environment Canada; 241-244.

Compagnucci RH, Salles MA, Canziani PO. 1999. Interannual variability of the $70 \mathrm{hPa}$ temperature in the Southern Hemisphere. In Preprints, 10th Symposiom on Global Change Studies, American Meteorological Society; 39-42.

Compagnucci RH, Salles MA, Canziani PO. 2001. The spatial and temporal behaviour of the lower stratospheric temperature over the Southern Hemisphere: the MSU view. Part I: data, methodology and temporal behaviour. International Journal of Climatology 21: 419-437.

Drowsdowsky W. 1993. Potential predictability of winter rainfall over Southern and Eastern Australia using Indian Ocean SST anomalies. Australian Meteorological Magazine 42: 1-6.

Graf H-F, Kirchner I, Perhvitz J. 1998. Changing lower circulation: the role of ozone and greenhouse gases. Journal of Geophysical Research 103: 11251-11262.

Huth R. 1993. An example of using obliquely rotated principal components to detect circulation types over Europe. Meteorologie Zeitschrift 2: 285-293.

North GR, Bell TL, Cahalan RF. 1982. Sampling errors in the empirical orthogonal functions. Monthly Weather Review 110: 699-706.

Preisendorfer RW. 1988. In Principal Component Analysis in Meteorology and Oceanography, Mobley CD (ed.). Elsevier: Amsterdam.

Ramaswamy V, Schwarzkopf MD, Randel W. 1996. Fingerprint of ozone depletion in the spatial and temporal pattern of recent lower-stratospheric cooling. Nature 382: 616-618.

Richman MB. 1983. Specification of the complex mode of circulation with T-mode factor analysis. In Preprints, Second International Conference on Statistics and Climate, Lisbon, 5.1.1. American Meteorological Society.

Richman MB, Gong X. 1999. Relationships between the hyperplane width to the fidelity of principal component loadings patterns. Journal of Climate 12: 1557-1576.

Tett SFB, Mitchell JFB, Parker DE, Allen MR. 1996. Human influence on the atmospheric vertical temperature structure: detection and observations. Science 274: 1170-1173.

Wirth V. 1991. Quasi-stationary planetary waves in total ozone and their correlation with lower stratospheric temperature, PhD Thesis, Ludwig-Maximilians Universitat, Muenchen. 